\title{
RATIONAL CURVES ON QUOTIENTS OF ABELIAN VARIETIES BY FINITE GROUPS
}

\author{
BO-HAE IM AND MICHAEL LARSEN
}

\begin{abstract}
In 3, it is proved that the quotient of an abelian variety $A$ by a finite order automorphism $g$ is uniruled if and only if some power of $g$ satisfies a numerical condition $0<\operatorname{age}\left(g^{k}\right)<1$. In this paper, we show that age $\left(g^{k}\right)=1$ is enough to guarantee that $A /\langle g\rangle$ has at least one rational curve.
\end{abstract}

\section{INTRODUCTION}

Let $G$ be a finite group of automorphisms of an abelian variety $A / \mathbb{C}$. It is a classical result [4, II. $\S 1$ ] that $A$ itself cannot contain a rational curve. For $|G|>1$, there may or may not be rational curves on $A / G$. For general abelian varieties, $\operatorname{Aut}(A)= \pm 1$, and Pirola proved [7] that for $A$ sufficiently general and of dimension at least three, $A / \pm 1$ has no rational curves. At the other extreme, regarding $A=E^{n}$ as the set of $n+1$-tuples of points on the elliptic curve $E$ which sum to $0, A / S_{n+1}$ can be interpreted as the set of effective divisors linearly equivalent to $(n+1)[0]$ and, as such, is just $\mathbb{P}^{n}$. More generally, Looijenga has shown [6] that the quotient of $E^{n}$ by the Weyl group of a root system of rank $n$ is a weighted projective space.

Rational curves on $A / G$ over a field $K$ are potentially a source of rational points over $G$-extensions of $K$. For instance, the method [5] for finding pairs $a, b \in \mathbb{Q}^{\times}$such that the quadratic twists $E_{a}, E_{b}$, and $E_{a b}$ all have positive rank amounts to finding a rational curve on $E^{3} /(\mathbb{Z} / 2 \mathbb{Z})^{2}$. Likewise, the theorem of Looijenga cited above gives for each elliptic curve $E$ over a number field $K$ and for each Weyl group $W$, a source of $W$-extensions $L_{i}$ of $K$ such that the representation of $W$ on each $E\left(L_{i}\right) \otimes \mathbb{Q}$ contains the reflection representation. On the other hand, the result of Pirola cited above dims the hope of using geometric methods to show that every abelian variety over a number field $K$ gains rank over infinitely many quadratic extensions of $K$. Thus, it is desirable from the viewpoint of arithmetic to understand when $A / G$ can be expected to have a rational curve over a given field $K$,

Date: September 17, 2018.

2000 Mathematics Subject Classification. 14K05.

Bo-Hae Im was partially supported by the National Research Foundation of Korea Grant funded by the Korean Government(MEST) (NRF-2011-0015557). Michael Larsen was partially supported by NSF grant DMS-1101424. 
and to begin with, one would like to know when $A / G$ has a rational curve over $\mathbb{C}$.

Any automorphism $g$ of an abelian variety $A$ defines an invertible linear transformation (also denoted $g$ ) on $\operatorname{Lie}(A)$. If $g$ is of finite order, there exists a unique sequence of rationals $0 \leq x_{1} \leq x_{2} \leq \cdots \leq x_{n}<1$ such that the eigenvalues of $g$ are $e\left(x_{1}\right), \ldots, e\left(x_{n}\right)$, where $e(x):=e^{2 \pi i x}$. We say $g$ is of type $\left(x_{1}, \ldots, x_{n}\right)$. Following Kollár and Larsen [3], we write age $(g)=x_{1}+\cdots+x_{n}$. For instance, age $(g)=1 / 2$ for every reflection $g$. The main result of [3] asserts that $A / G$ is uniruled if and only if $0<\operatorname{age}(g)<1$ for some $g \in G$. In this paper, we prove that to find a single rational curve in $A / G$, it sufices that age $(g) \leq 1$.

Since we need only consider the case age $(g)=1$, we first classify all types of weight 1 . This requires a combinatorial analysis, which we carried out using a computer algebra system to minimize the risk of an oversight. There are thirty-five cases (see Table 2 below), and our strategy for finding rational curves depends on case analysis. Abelian surfaces play a special role, since here we can use known results on K3 surfaces. The other key idea is to find a non-singular projective curve $X$ on which $G$ acts with quotient $\mathbb{P}^{1}$ and a $G$-equivariant map from $X$ to $A$, or, equivalently, a $G$-homomorphism from the Jacobian variety of $X$ to $A$.

We would like to thank Yuri Tschinkel and Alessio Corti for helpful comments on earlier versions of this paper.

\section{Classifying types}

If $A=V / \Lambda$, then the Hodge decomposition $\Lambda \otimes \mathbb{C} \cong V \oplus \bar{V}$ respects the action of $\operatorname{Aut}(A)$. Therefore, if $g$ is of finite order with eigenvalues $e\left(x_{1}\right), \ldots, e\left(x_{n}\right)$, then the multiset

$$
\left\{e\left(x_{1}\right), \ldots, e\left(x_{n}\right), e\left(-x_{1}\right), \ldots, e\left(-x_{n}\right)\right\}
$$

is $\operatorname{Aut}(\mathbb{C})$-stable. By a type, we mean a multiset $\left\{x_{1}, \ldots, x_{n}\right\}$ with $x_{i} \in$ $[0,1)$ such that the multiset $(*)$ is $\operatorname{Aut}(\mathbb{C})$-stable. Equivalently, a type can be identified with a finitely supported function $f: \mathbb{Q} / \mathbb{Z} \rightarrow \mathbb{N}$ such that $f(x)+f(-x)$ depends only on the order of $x$ in $\mathbb{Q} / \mathbb{Z}$. By the weight of $\left\{x_{1}, \ldots, x_{n}\right\}$, we mean the sum $x_{1}+\cdots+x_{n}$, so that age $(g)$ is the weight of the type of $g$.

A type is reduced if 0 does not appear, and the reduced type of a given type is obtained by discarding all copies of 0 . The sum of types is the union in the sense of multisets; at the level of associated functions on $\mathbb{Q} / \mathbb{Z}$ it is the usual sum. A type which is not the sum of non-zero types is primitive. All the elements of a primitive type appear with multiplicity one, and they all have the same denominator. Every type can be realized (not necessarily uniquely) as a sum of primitive types; if the weight of the type is 1 , each of the primitive types has weight $\leq 1$, so our first task is to classify primitive types with weight $\leq 1$. 
A primitive type $X$ of denominator $n \geq 2$ consists of fractions $a_{i} / n$ where $0<a_{i}<n$, and $\left(a_{i}, n\right)=1$. Moveover, if $n \geq 3$, for each positive integer $a<n$ prime to $n$, exactly one of $a / n$ and $1-a / n$ belongs to $X$. If $a<b<n / 2$ and $1-a / n \in X$, then the weight of $X$ exceeds 1 since either $b / n$ or $1-b / n$ belongs to $X$. Thus, if $0<a<n / 2$ and $1-a / n \in X$, then $a$ must be the largest integer in $(0, n / 2)$ prime to $n$.

Lemma 1. If $\phi(n)>24$, then

$$
\sum_{x \in S_{n}} \min (x, n-x)>2 n,
$$

where $S_{n}$ is the set of positive integers $<n$ and prime to $n$. Moreover, the largest integer $n$ such that $\phi(n) \leq 24$ is 90 .

Proof. Note that

$$
\min (x, n-x)>\frac{x(n-x)}{n}=\frac{n^{2}-x^{2}-(n-x)^{2}}{2 n} .
$$

In order to prove the first statement, we want to prove if $\phi(n)>24$, then

$$
\sum_{x \in S_{n}}\left(n^{2}-x^{2}-(n-x)^{2}\right)>4 n^{2}
$$

or equivalently,

$$
\phi(n) n^{2}-2 \sum_{x \in S_{n}} x^{2}-4 n^{2}>0 .
$$

By Möbius inversion, one can prove that

$$
\sum_{x \in S_{n}} x^{2}=\frac{\phi(n) n^{2}}{3}+(-1)^{d_{n}} \frac{\phi(f(n)) n}{6},
$$

where $f(n)$ denotes the larges squarefree divisor of $n$ and $d_{n}$ is the number of distinct prime divisors of $n$. Thus, if $\phi(n)>24$, then $\phi(n)>24 \geq 12 \frac{n}{n-1}$, so $(n-1) \phi(n)-12 n>0$ and since $\phi(f(n)) \leq \phi(n)$,

$$
\begin{aligned}
\phi(n) n^{2}-2 \sum_{x \in S_{n}} x^{2}-4 n^{2} & \geq\left(\frac{\phi(n)}{3}-4\right) n^{2}-\frac{\phi(n) n}{3} \\
& =\frac{n((n-1) \phi(n)-12 n)}{3}>0,
\end{aligned}
$$

which is the desired inequality.

For the second statement, if $\phi(n) \leq 24$ and $p$ is a prime factor of $n$, then $\phi(p)=p-1 \leq \phi(n) \leq 24$. Hence $p \leq 23$. Writing

$$
n=2^{n_{2}} 3^{n_{3}} 5^{n_{5}} 7^{n_{7}} 11^{n_{11}} 13^{n_{13}} 17^{n_{17}} 19^{n_{19}} 23^{n_{23}},
$$

we have

$$
0 \leq n_{2} \leq 5,0 \leq n_{3} \leq 3,0 \leq n_{5} \leq 2,
$$

and $0 \leq n_{i} \leq 1$ for $7 \leq i \leq 23$. Case analysis now shows $n \leq 90$. 
Proposition 2. There are 28 primitive types with weight $\leq 1$ :

\begin{tabular}{|l|c|c|c|}
\hline$\#$ & $\mathrm{n}$ & primitive types & weight \\
\hline 1 & 2 & $1 / 2$ & $1 / 2$ \\
\hline 2 & 3 & $1 / 3$ & $1 / 3$ \\
3 & & $2 / 3$ & $2 / 3$ \\
\hline 4 & 4 & $1 / 4$ & $1 / 4$ \\
5 & & $3 / 4$ & $3 / 4$ \\
\hline 6 & 5 & $1 / 5,2 / 5$ & $3 / 5$ \\
7 & & $1 / 5,3 / 5$ & $4 / 5$ \\
\hline 8 & 6 & $1 / 6$ & $1 / 6$ \\
9 & & $5 / 6$ & $5 / 6$ \\
\hline 10 & 7 & $1 / 7,2 / 7,3 / 7$ & $6 / 7$ \\
11 & & $1 / 7,2 / 7,4 / 7$ & 1 \\
\hline 12 & 8 & $1 / 8,3 / 8$ & $1 / 2$ \\
13 & & $1 / 8,5 / 8$ & $3 / 4$ \\
\hline 14 & 9 & $1 / 9,2 / 9,4 / 9$ & $7 / 9$ \\
15 & & $1 / 9,2 / 9,5 / 9$ & $8 / 9$ \\
\hline 16 & 10 & $1 / 10,3 / 10$ & $2 / 5$ \\
17 & & $1 / 10,7 / 10$ & $4 / 5$ \\
\hline 18 & 12 & $1 / 12,5 / 12$ & $1 / 2$ \\
19 & & $1 / 12,7 / 12$ & $2 / 3$ \\
\hline 20 & 14 & $1 / 14,3 / 14,5 / 14$ & $9 / 14$ \\
21 & & $1 / 14,3 / 14,9 / 14$ & $13 / 14$ \\
\hline 22 & 15 & $1 / 15,2 / 15,4 / 15,7 / 15$ & $14 / 15$ \\
23 & & $1 / 15,2 / 15,4 / 15,8 / 15$ & 1 \\
\hline 24 & 16 & $1 / 16,3 / 16,5 / 16,7 / 16$ & 1 \\
\hline 25 & 18 & $1 / 18,5 / 18,7 / 18$ & $13 / 18$ \\
26 & & $1 / 18,5 / 18,11 / 18$ & $17 / 18$ \\
\hline 27 & 20 & $1 / 20,3 / 20,7 / 20,9 / 20$ & 1 \\
\hline 28 & 24 & $1 / 24,5 / 24,7 / 24,11 / 24$ & 1 \\
\hline & & 1961 & \\
& & & \\
\hline
\end{tabular}

Table 1

Proof. For $n \geq 3$, the weight of a primitive type of denominator $n$ is at least

$$
\sum_{\left\{x \in S_{n} \mid x<n / 2\right\}} \frac{x}{n} \geq \frac{1}{2 n} \sum_{x \in S_{n}} \min (x, n-x) .
$$

By Lemma 1, it suffices to carry out an exhaustive search up to $n=90$.

Lemma 3. There are 35 types with age 1 of automorphisms given in Table 2 below. 


\begin{tabular}{|c|c|c|c|}
\hline$\#$ & $\mathrm{n}$ & types & notes \\
\hline 1 & 2 & $1 / 2,1 / 2$ & Prop. 6] \\
\hline 2 & 3 & $1 / 3,1 / 3,1 / 3$ & Th. 12 \\
\hline 3 & & $1 / 3,2 / 3$ & Prop. 7 \\
\hline 4 & 4 & $1 / 4,1 / 4,1 / 4,1 / 4$ & Th. 12 \\
\hline 5 & & $1 / 4,1 / 4,2 / 4$ & $g^{2} \rightarrow \# 1$ \\
\hline 6 & & $1 / 4,3 / 4$ & $g^{2} \rightarrow \# 1$ \\
\hline 7 & 6 & $1 / 6,1 / 6,1 / 6,1 / 6,1 / 6,1 / 6$ & Th. 12 \\
\hline 8 & & $1 / 6,1 / 6,1 / 6,1 / 6,2 / 6$ & Th. 12 \\
\hline 9 & & $1 / 6,1 / 6,1 / 6,3 / 6$ & $g^{2} \rightarrow \# 2$ \\
\hline 10 & & $1 / 6,1 / 6,4 / 6$ & $g^{3} \rightarrow \# 1$ \\
\hline 11 & & $1 / 6,5 / 6$ & $g^{3} \rightarrow \# 1$ \\
\hline 12 & & $1 / 6,2 / 6,3 / 6$ & $g^{3} \rightarrow \# 1$ \\
\hline 13 & & $1 / 6,1 / 6,2 / 6,2 / 6$ & $g^{3} \rightarrow \# 1$ \\
\hline 14 & 7 & $1 / 7,2 / 7,4 / 7$ & Cor. 10 \\
\hline 15 & 8 & $1 / 8,2 / 8,5 / 8$ & $g^{4} \rightarrow \# 1$ \\
\hline 16 & & $1 / 8,3 / 8,4 / 8$ & $g^{4} \rightarrow \# 1$ \\
\hline 17 & & $1 / 8,1 / 8,3 / 8,3 / 8$ & Th. 12 \\
\hline 18 & & $1 / 8,2 / 8,2 / 8,3 / 8$ & $g^{4} \rightarrow \# 1$ \\
\hline 19 & 10 & $1 / 10,2 / 10,3 / 10,4 / 10$ & $g^{5} \rightarrow \# 1$ \\
\hline 20 & 12 & $4 / 12,2 / 12,1 / 12,5 / 12$ & $g^{6} \rightarrow \# 1$ \\
\hline 21 & & $4 / 12,3 / 12,3 / 12,2 / 12$ & $g^{6} \rightarrow \# 1$ \\
\hline 22 & & $6 / 12,1 / 12,5 / 12$ & $g^{6} \rightarrow \# 1$ \\
\hline 23 & & $3 / 12,3 / 12,1 / 12,5 / 12$ & $g^{4} \rightarrow \# 3$ \\
\hline 24 & & $2 / 12,2 / 12,2 / 12,1 / 12,5 / 12$ & $g^{6} \rightarrow \# 1$ \\
\hline 25 & & $1 / 12,1 / 12,5 / 12,5 / 12$ & Th. 12 \\
\hline 26 & & $4 / 12,1 / 12,7 / 12$ & $g^{6} \rightarrow \# 1$ \\
\hline 27 & & $2 / 12,2 / 12,1 / 12,7 / 12$ & $g^{6} \rightarrow \# 1$ \\
\hline 28 & & $3 / 12,3 / 12,2 / 12,2 / 12,2 / 12$ & $g^{6} \rightarrow \# 1$ \\
\hline 29 & 15 & $1 / 15,2 / 15,4 / 15,8 / 15$ & Cor. 11 \\
\hline 30 & 16 & $1 / 16,3 / 16,5 / 16,7 / 16$ & Cor. 9] \\
\hline 31 & 20 & $1 / 20,3 / 20,7 / 20,9 / 20$ & Cor. 9] \\
\hline 32 & 24 & $1 / 24,5 / 24,7 / 24,11 / 24$ & Cor. 9 \\
\hline 33 & & $8 / 24,4 / 24,3 / 24,9 / 24$ & $g^{12} \rightarrow \# 1$ \\
\hline 34 & & $3 / 24,9 / 24,2 / 24,10 / 24$ & $g^{12} \rightarrow \# 1$ \\
\hline 35 & & $4 / 24,4 / 24,4 / 24,3 / 24,9 / 24$ & $g^{12} \rightarrow \# 1$ \\
\hline
\end{tabular}

Table 2

Proof. Let $\left[a_{i}\right]$ be a formal variable representing the $i$ th primitive type in Table 1, and let $w_{i}$ denote the weight of the $i$ th type. A monomial $\prod a_{i}^{m_{i}}$ stands for a sum of primitive types in which the $i$ th type appears $m_{i}$ times. The g.c.d. of the denominators of the $w_{i}$ is 5040 . Let $y=x^{1 / 5040}$, so $\left(1-\left[a_{i}\right] x^{w_{i}}\right)^{-1}$ is a power series in $y$ for every $i$. By MAPLE 13, the coefficient 
of $y^{5040}$ in the product

$$
\prod_{i=1}^{28}\left(1-\left[a_{i}\right] x^{w_{i}}\right)^{-1}=\prod_{i=1}^{28}\left(1-\left[a_{i}\right] y^{w_{i} \cdot 5040}\right)^{-1},
$$

is $a 1^{2}+a 2^{3}+a 2 a 3+a 4 a 5+a 8 a 9+a 4 a 13+a 6 a 16+a 4^{2} a 1+a 28+a 27+$ $a 23+a 24+a 11+a 19 a 2+a 19 a 8^{2}+a 18 a 1+a 18 a 4^{2}+a 18 a 8^{3}+a 18 a 12+$ $a 18^{2}+a 12 a 1+a 12 a 4^{2}+a 12 a 8^{3}+a 12^{2}+a 8^{2} a 2^{2}+a 8^{2} a 3+a 8^{3} a 1+$ $a 8^{3} a 4^{2}+a 8^{4} a 2+a 8^{6}+a 4^{4}+a 18 a 8 a 2+a 12 a 8 a 2+a 8 a 1 a 2+a 8 a 2 a_{4}{ }^{2}$.

Each monomial in this sum corresponds to an entry in Table 2.

\section{Rational Curves in $A /\langle g\rangle$}

In this section we explain how to find rational curves on $A /\langle g\rangle$ in each case in Table 2 .

Lemma 4. If $A /\left\langle g^{n}\right\rangle$ has a rational curve for some positive integer $n$, then $A /\langle g\rangle$ has a rational curve.

Proof. The morphism $A /\left\langle g^{n}\right\rangle \rightarrow A /\langle g\rangle$ is finite, so the image of a rational curve is again a rational curve.

Proposition 5. Let $A$ be an abelian variety and $g$ an automorphism of finite order. Suppose that for every abelian variety $B$ and finite-order automorphism $h \in \operatorname{Aut}(B)$ whose type is the reduced type of $g, B /\langle h\rangle$ has a rational curve. Then $A /\langle g\rangle$ has a rational curve.

Proof. Let $B$ denote the image of $1-g$ acting on $A$. Then $B$ is an abelian subvariety of $A$, and $g$ restricts to an automorphism $h$ of $B$ whose type is the reduced type of $g$. As $B /\langle h\rangle \subset A /\langle g\rangle$ has a rational curve, the same is true of $A /\langle g\rangle$.

The following proposition is well known.

Proposition 6. If $A$ is an abelian surface, then $A / \pm 1$ has a rational curve.

Proof. Resolving the 16 singularities of $A / \pm 1$, we obtain a K3 surface with Picard number $\geq 16 \geq 5$. By work of Bogomolov and Tschinkel [1], any such surface is either elliptic or has infinite automorphism group and in either case has infinitely many rational curves, all but finitely many of which lie on $A / \pm 1$.

Note that Proposition 6 covers not only case \#1 in Table 2 but twenty other cases as well, namely those (indicated in the "notes" column) for which the reduced type of some power of $g$ is $(1 / 2,1 / 2)$.

Proposition 7. Let $A \cong V / \Lambda$ be an abelian surface with an automorphism $g$ of type $(1 / 3,2 / 3)$. Then $A /\langle g\rangle$ contains a rational curve. 
Proof. Let $G=\langle g\rangle$ and $X=A / G$. Regarding $1-g$ as an isogeny of $A$, the number of fixed points of $g$ is

$$
\operatorname{deg}(1-g)=\# \operatorname{ker}(1-g)=\operatorname{det}(1-g \mid \Lambda)=3^{2}=9
$$

These are singularities of type $A_{2}$, since under $(x, y) \mapsto\left(\omega x, \omega^{2} y\right)$ where $\omega$ is a cube root of unity, the invariants are generated by $X=x^{3}, Y=y^{3}, Z=x y$, and so

$$
\mathbb{C}[[x, y]]^{G}=\mathbb{C}[[X, Y, Z]] /\left(X Y-Z^{3}\right) .
$$

This is isomorphic to $\mathbb{C}[[x, y, z]] /\left(x^{2}+y^{2}+z^{3}\right)$, which has a Du Val singularity of type $A_{2}$ (see $[8$, Ch.4, 4.2]).

Consider the minimal resolution $f: Y \rightarrow X$, for which the 9 exceptional divisors $Y_{i}$ each consists of two projective lines $D_{i, 1}$ and $D_{i, 2}$ intersecting at one point. The canonical divisor of $Y$ is $K_{Y}=f^{*} K_{X}=0$. Hence $Y$ is a K3-surface of Picard number $\geq 18$, and again by [1], we deduce that $X$ has infinitely many rational curves..

Theorem 8. Let $B$ be an abelian variety and $h \in \operatorname{Aut}(B)$ an automorphism of finite order such that $h$ and $h^{-1}$ have disjoint types. Let $A$ be an abelian variety with a finite order automorphism $g$ whose type is contained in that of $h$. If there is a rational curve on $B /\langle h\rangle$ then there is a rational curve on $A /\langle g\rangle$.

Proof. It suffices to prove that there exists a surjective homomorphism $p: B \rightarrow A$ such that the diagram

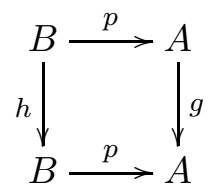

commutes. Writing $B=\operatorname{Lie}(B) / \Lambda_{B}$ and $A=\operatorname{Lie}(A) / \Lambda_{A}$, the goal is to find a surjective $\mathbb{C}[t]$-linear map $\phi: \operatorname{Lie}(B) \rightarrow \operatorname{Lie}(A)$ (where $t$ acts as $g$ on $\operatorname{Lie}(A)$ and as $h$ on $\operatorname{Lie}(B))$ such that $\phi\left(\Lambda_{B}\right) \subset \Lambda_{A}$. If $\psi$ is a surjective $\mathbb{C}[t]$-linear map $\operatorname{Lie}(B) \rightarrow \operatorname{Lie}(A)$ such that $\psi\left(\Lambda_{B} \otimes \mathbb{Q}\right)=\Lambda_{A} \otimes \mathbb{Q}$, then we can define $\phi:=n \psi$ for $n$ a sufficiently divisible positive integer. It suffices, therefore, to find $\psi$ with the desired properties.

As the type of $A$ is a subset of the type of $B$, there exists a surjective $\mathbb{Q}[t]$-linear map $T: \Lambda_{B} \otimes \mathbb{Q} \rightarrow \Lambda_{A} \otimes \mathbb{Q}$. Extending scalars to $\mathbb{C}, T \otimes 1$ maps $\Lambda_{B} \otimes \mathbb{C}=\operatorname{Lie}(B) \oplus \overline{\operatorname{Lie}(B)}$ to $\Lambda_{A} \otimes \mathbb{C}=\operatorname{Lie}(A) \oplus \overline{\operatorname{Lie}(A)}$. The type of $g$ acting on $\overline{\operatorname{Lie}(A)}$ is the same as the type of $g^{-1}$ acting on $\operatorname{Lie}(A)$ and therefore disjoint from the type of $g$ acting on $\operatorname{Lie}(A)$, and the same is true for the type of $h$ acting on $\operatorname{Lie}(B)$. As $\operatorname{Lie}(B)$ and $\operatorname{Lie}(A)$ are direct sums of certain $t$-eigenspaces of $\Lambda_{B} \otimes \mathbb{C}$ and $\Lambda_{A} \otimes \mathbb{C}$ respectively and as the spectrum of $t$ acting on $\operatorname{Lie}(A)$ is the intersection of the spectra of $t$ acting on $\operatorname{Lie}(B)$ and on $\Lambda_{A} \otimes \mathbb{C}$, it follows that $T \otimes 1$ maps $\operatorname{Lie}(B)$ to $\operatorname{Lie}(A)$. The restriction of $\psi$ to $\operatorname{Lie}(B)$ is therefore the desired map. 
Corollary 9. Let $n \geq 3$ be a positive integer, and let $m=\lceil n / 2\rceil-1$. If $A$ is an abelian variety and $g \in \operatorname{Aut}(A)$ is an automorphism of order $n$ whose type is contained in $\{1 / n, 2 / n, \ldots, m / n\}$, then $A /\langle g\rangle$ has a rational curve.

Proof. Let $X$ denote the non-singular projective hyperelliptic curve of genus $m$ which contains the affine curve $y^{2}=x^{n}-1$. The order- $n$ automorphism $h(x, y)=(e(1 / n) x, y)$ extends to an automorphism of $X$ and therefore defines an automorphism of $B:=\operatorname{Jac}(X)$. The Lie algebra $\operatorname{Lie}(B)$ can be identified with the space $H^{0}\left(X, \Omega_{X}\right)$ of holomorphic differential forms on $X$, which has a basis

$$
\left\{\frac{d x}{y}, \frac{x d x}{y}, \ldots, \frac{x^{m-1} d x}{y}\right\}
$$

Therefore, the type of $h$ acting on $B$ is $\{1 / n, 2 / n, \ldots, m / n\}$, which is disjoint from the type of $h^{-1}$. On the other hand, $B /\langle h\rangle$ contains the rational curve $X /\langle h\rangle$. Thus, Theorem 8 applies.

Corollary 10. If $A$ is an abelian 3-fold and $g$ is an automorphism of $A$ of type $(1 / 7,2 / 7,4 / 7)$, then $A /\langle g\rangle$ has a rational curve.

Proof. Let $X$ denote the Klein quartic:

$$
X: x^{3} y+y^{3} z+z^{3} x=0
$$

and $B$ the Jacobian of $X$. The self-map $h(x, y, z)=\left(\zeta_{7} x, \zeta_{7}^{4} y, \zeta_{7}^{2} z\right)$ of $X$ belongs to the automorphism group $\mathrm{PSL}_{2}\left(\mathbb{F}_{7}\right)$ of $X$ which acts non-trivially on the Jacobian variety $B$ and therefore on $\operatorname{Lie}(B)=H^{0}\left(X, \Omega_{X}\right)$. Conjugating $h$ by the cyclic permutations of $(x, y, z)$, we see that $h$ is conjugate to $h^{2}$ and $h^{4}$ in $\operatorname{Aut}(X)$, and therefore the type of $h$ is invariant under multiplication by $2(\bmod 1)$. It is therefore $(1 / 7,2 / 7,4 / 7)$ or $(3 / 7,5 / 7,6 / 7)$, and replacing $h$ by $h^{-1}$ if necessary, we may assume that it is the former.

We remark that $B /\langle h\rangle$ in the proof of Corollary 10 has appeared in the literature; it is known to have a Calabi-Yau resolution [2, Example 6.3].

Corollary 11. If $A$ is an abelian variety and $g$ an automorphism such that the type of $A$ is contained in $(1 / 15,2 / 15,3 / 15,4 / 15,8 / 15,9 / 15)$, then $A /\langle g\rangle$ has a rational curve.

Proof. Let $X$ be the non-singular projective curve which has a (singular, affine) model $X^{\prime}: y^{15}=x^{2}(x-1)$. This is singular only at $(0,0)$, and the inverse image of this singularity under the normalization map $X \backslash\left\{P_{\infty}\right\} \rightarrow$ $X^{\prime}$ is a single point $P_{0} \in X$. The automorphism $h:(x, y) \mapsto(x, e(1 / 15) y)$ of the affine curve induces an automorphism of $X$ of order 15 . As $15 y^{14} d y=$ $\left(3 x^{2}-2 x\right) d x$, any differential form $\frac{x^{m} y^{n} d y}{3 x^{2}-2 x}, m, n \geq 0$, is holomorphic except possibly at $P_{0}$ and $P_{\infty}$. One checks that

$$
\frac{d y}{3 x-2}, \frac{y d y}{3 x-2}, \frac{y^{2} d y}{3 x-2}, \frac{y^{3} d y}{3 x-2}, \frac{y^{7} d y}{3 x^{2}-2 x}, \frac{y^{8} d y}{3 x^{2}-2 x}
$$


are all holomorphic, and their eigenvalues under $h$ are $e(1 / 15), e(2 / 15)$, $e(3 / 15), e(4 / 15), e(8 / 15), e(9 / 15)$ respectively. Applying the RiemannHurwitz theorem to the map $X \rightarrow \mathbb{P}^{1}$ given by $y$, we see that $X$ is of genus 6 , and therefore, that these differential forms form a basis of $\operatorname{Lie}(\operatorname{Jac}(B))=$ $H^{0}\left(X, \Omega_{X}\right)$.

Theorem 12. If $A$ is an abelian variety and $g$ is an automorphism of finite order such that $g$ and $g^{-1}$ have disjoint types and the type of $g$ is a sum of primitive types at least one of which has weight less than 1 , then $A /\langle g\rangle$ has a rational curve.

Proof. For every primitive type, there exists an abelian variety $B_{i}$ with complex multiplication and an automorphism $h_{i}$ of $B_{i}$ with the given type. Indeed, the primitive types of denominator $n$ are in natural correspondence with CM-types on $\mathbb{Q}\left(\zeta_{n}\right)=\mathbb{Q}(e(1 / n))$. Any CM-type $\Phi$ on $\mathbb{Q}\left(\zeta_{n}\right), n \geq 3$ defines an embedding $\mathbb{Q}\left(\zeta_{n}\right) \rightarrow \mathbb{C}^{\phi(n) / 2}$. The image of $\mathbb{Z}\left[\zeta_{n}\right]$ defines a lattice $\Lambda \subset \mathbb{C}^{\phi(n) / 2}$, and the quotient $\mathbb{C}^{\phi(n) / 2} / \Lambda$ is a complex torus with a natural action of $\mathbb{Z} / n \mathbb{Z}$ of the type associated with $\Phi$. The quotient $\mathbb{C}^{\phi(n) / 2} / \Lambda$ admits a polarization [9, II 6 Theorem 4], so there exists a pair $\left(B_{i}, h_{i}\right)$ as claimed. If age $\left(h_{1}\right)<1$, then by [3], $B_{1} /\left\langle g_{1}\right\rangle$ has a rational curve. If $A=A_{1} \times \cdots \times A_{m}$, and $g=\left(g_{1}, \ldots, g_{m}\right)$ is a finite order automorphism of $A$ which stabilizes each factor, then $A_{1} /\left\langle g_{1}\right\rangle \subset A /\langle g\rangle$, so $A /\langle g\rangle$ has a rational curve. The theorem now follows from Theorem 8 .

To summarize, we have the following theorem:

Theorem 13. Let $A$ be an abelian variety with a nontrivial automorphism $g$ of finite order such that age $(g) \leq 1$. Then $A /\langle g\rangle$ contains a rational curve.

Corollary 14. Let $A$ be an abelian variety of dimension $n$ with a nontrivial automorphism $g$ of finite order. If $\operatorname{dim}(\operatorname{ker}(1-g)) \geq n-2$ (i.e. the codimension of the fixed subspace of $A$ under $g$ is less than or equal to 2), then the quotient $A /\langle g\rangle$ contains a uniruled hypersurface.

Proof. Since $\operatorname{dim}(\operatorname{ker}(1-g)) \geq n-2, B:=\operatorname{im}(1-g)$ is an abelian variety of dimension $n-\operatorname{dim}(\operatorname{ker}(1-g)) \leq 2$. Let $h$ denote the restriction of $g$ to $B$. As age $(h)+\operatorname{age}\left(h^{-1}\right) \geq 2$, we may assume without loss of generality that age $(h) \leq 1$, so $B /\langle h\rangle$ has a rational curve $Z$ by Theorem 13. Let $C$ denote the identity component of $\operatorname{ker}(1-g)$, which is an abelian subvariety of dimension dim $\operatorname{ker}(1-g)$ on which $g$ acts trivially. The addition morphism $B \times C \rightarrow A$ is an isogeny and respects the action of $\langle g\rangle$. We therefore obtain a finite morphism from

$$
Z \times C \subset(B /\langle g\rangle) \times C \cong(B \times C) /\langle g\rangle
$$

to $A /\langle g\rangle$. The image of an $n-1$-dimensional ruled variety under a finite morphism is a uniruled hypersurface. 
Corollary 15. Let $E$ be an elliptic curve. If $W$ is a Weyl group of simple roots of rank $n \geq 3$ acting on $E^{n}$ and $W^{+}$is an index 2-subgroup of $W$, then the quotient $E^{n} / W^{+}$contains a rational curve.

Proof. $W$ is generated by reflections $s_{j}$ of simple roots. Since $W^{+}$is an index 2 -subgroup of $W$, there exist two reflections $s_{1}$ and $s_{2}$ such that $s_{1} s_{2} \in W^{+}$. Then for each $i, \operatorname{ker}\left(1-s_{i}\right)$ has codimension 1 and their intersection has codimension $\leq 2$. Since $\operatorname{ker}\left(1-s_{1} s_{2}\right)$ contains the intersection of $\operatorname{ker}\left(1-s_{1}\right)$ and $\operatorname{ker}\left(1-s_{2}\right)$, this follows from Corollary 14 .

\section{REFERENCES}

[1] Bogomolov, Fedor; Tschinkel, Yuri: Density of rational points on elliptic K3 surfaces, Asian J. Math. 4, (2000), 351-368,

[2] Bogomolov, Fedor; Tschinkel, Yuri: Rational curves and points on K3 surfaces. Amer. J. Math. 127 (2005), no. 4, 825-835.

[3] Kollár, János; Larsen, Michael: Quotients of Calabi-Yau varieties. Algebra, arithmetic, and geometry: in honor of Yu. I. Manin. Vol. II, 179-211, Progr. Math., 270, Birkhauser Boston, Inc., Boston, MA, 2009.

[4] Lang, Serge: Abelian varieties, New York: Springer-Verlag, 1983.

[5] Larsen, Michael: Rank of elliptic curves over almost algebraically closed fields. Bull. London Math. Soc. 35 (2003), no. 6, 817-820.

[6] Looijenga, Eduard: Root systems and elliptic curves. Invent. Math. 38 (1976/77), no. $1,17-32$.

[7] Pirola, Gian Pietro: Curves on generic Kummer varieties. Duke Math. J. 59 (1989), no. 3, 701-708.

[8] Reid, Miles: Chapters on algebraic surfaces, Complex algebraic geometry (Park City, UT, 1993), 3-159, IAS/Park City Math. Ser., 3, Amer. Math. Soc., Providence, RI, 1997.

[9] Shimura, Goro: Abelian Varieties with Complex Multiplication and Modular Functions. Princeton Mathematical Series, 46. Princeton University Press, Princeton, NJ, 1998.

Department of Mathematics, Chung-Ang University, 221, Heukseok-dong, Donguak-Gu, Seoul, 156-756, South Korea

E-mail address: bohaeim@gmail.com, imbh@cau.ac.kr

Department of Mathematics, Indiana University, Bloomington, Indiana 47405, USA

E-mail address: mjlarsen@indiana.edu 